\title{
Entrenamiento mental breve en adolescentes con trastorno alimentario no especificado que presentan hábitos exigentes de actividad física
}

\author{
Brief mental training in adolescents suffering from a non-specific eating disorder who have demanding habits of \\ physical activity
}

\author{
Pablo de la Cerda \\ Facultad de Psicología, Universidad Santo Tomás, Chile \\ pdelacerda@hotmail.com
}

Daniel Mayorga-Vega

Departamento de Didáctica de la Expresión Musical, Plástica y Corporal, Universidad de Jaén, España

dmayorgavega@gmail.com

\section{Jesús Viciana}

Departamento de Educación Física y Deportiva, Universidad de Granada, España

jviciana@ugr.es

\section{RESUMEN:}

El objetivo principal del presente estudio fue examinar el efecto de un programa de entrenamiento mental breve sobre el estado afectivo, las actitudes hacia la alimentación y los niveles de actividad física en adolescentes diagnosticadas con Trastorno Alimentario No Especificado que practicaban una rutina exigente de actividad física. Una muestra de ocho mujeres adolescentes chilenas de 15-17 años de edad realizó un programa de entrenamiento mental breve durante ocho semanas una sesión por semana: 1) Conocimiento y establecimiento de objetivos; 2) Focalización y toma de conciencia mental y corporal; 3) Autocontrol psicobiológico; 4) Reposicionamiento del locus de control; 5) Autogestión y control de los pensamientos; 6-7) Re-focalización y reencuadre motivacional, y 8) Generalización y enfoque responsable de la voluntad. El Inventario de Depresión de Beck, el Cuestionario de Actitudes ante la Alimentación EAT-26 y una entrevista breve sobre los niveles de práctica y motivos de actividad física, se aplicaron antes y después del programa. Los resultados reflejan una influencia favorable del programa en la mejora del estado afectivo, de las actitudes hacia la alimentación y hacia la actividad física en las adolescentes intervenidas. Un programa de entrenamiento mental breve sería recomendable como complemento terapéutico en adolescentes con tales características.

Palabras Clave: Salud mental, Trastornos de la conducta alimentaria, Adolescencia, Jóvenes, Ejercicio físico.

\section{Abstract:}

The main purpose of the present study was to examine the effect of a brief mental training program on the emotional condition, attitudes toward alimentation and physical activity levels in adolescents suffering from a Non Specific Eating Disorder who have demanding habits of physical activity. A sample of eight Chilean female adolescents aged 15-17 years performed a brief mental training program for eight weeks one session per week: 1) Knowledge and establishment of objectives; 2) Focus and awareness of mind and body; 3) Psychobiological self-control; 4) Repositioning of the control locus; 5) Self-management and control of thoughts; 6-7) Refocusing and reframing of motivation, and 8) Generalization and responsible approach to the will. The Beck Depression Inventory, the Eating Attitudes Test EAT-26 and a brief interview about the practise levels and reasons of physical activity were applied before and after the program. The results confirmed the effectiveness of the mental training program on the emotional condition and attitudes toward alimentation and physical activity in the female adolescents intervened. A brief mental training program could be recommended as a therapeutic compliment in adolescents with these characteristics.

KEYWORDS: Mental health, Eating disorders, Adolescence, Young people, Exercise. 


\section{INTRODUCCIÓN}

La explosión de los medios de comunicación masivos, la emergencia de las redes sociales y la comunicación virtual han posibilitado que una gran cantidad de estímulos, modelos y tendencias hayan penetrado en las necesidades de la población con edades cada vez más precoces. Los hábitos de alimentación son un claro ejemplo de esta influencia. La gran variedad de productos alimenticios, la instalación excesiva de servicios de alimentación en centros comerciales, y el posicionamiento de las cadenas de comida rápida propician un desorden nutricional caracterizado por una saturación de grasas y altos niveles de azúcares. Por otro lado, una alimentación adecuada es aquella que consigue un equilibrio entre lo que el organismo necesita y lo que ingiere, dotándolo de los nutrientes necesarios para desempeñarse bien (Delgado, Gutiérrez, \& Castillo, 2004).

Durante la segunda infancia y principalmente con la llegada de la pubertad, el organismo consume gran cantidad de energía, que preparará al individuo para posteriormente ejercer su función reproductiva y otras propias de la adultez. Es normal que en este período aumente el apetito y la necesidad de ingerir los nutrientes necesarios para la maduración y crecimiento. Según la Organización Mundial de la Salud (Vásquez, de Cos, \& López-Nomdedeu, 2005) más de mil millones de personas en el mundo tienen sobrepeso, de las cuales 300 millones son obesas. En Chile las estadísticas muestran un crecimiento sostenido a través de los años: el índice de sobrepeso y obesidad entre los siete y nueve años es de un 35\%, y de un 20\% entre los 10 y 19 años (Ministerio de Salud, 2005). Por otro lado, la búsqueda de identidad y el proceso de individualización propios de la pubertad aumentan las inseguridades en el joven, donde el factor imagen corporal es muy relevante en su autoaceptación y autoestima. Harter (2003) describió un perfil de autopercepción en la adolescencia, donde para el desarrollo de la autoestima destaca la apariencia física por encima de la aceptación social o la competencia académica.

En la sociedad occidental se ha asociado el éxito personal, entre otras cosas, con la consecución de una imagen corporal que resalta la delgadez (Behar, 1996). Los medios de comunicación y la publicidad, la destaca imponiendo cánones consensuados de éxito (más concretamente de belleza) que distan bastante de la media ponderal esperable para ciertos rangos de edad, los cuales muchas veces se obtienen producto de estrictas exigencias y restricciones o por métodos artificiales. Es así como los jóvenes que más se alejan de estos cánones suelen ser sujetos de discriminación u hostilización por parte de sus pares, provocando insatisfacción y frustración. Dentro de la gama de alternativas de respuesta del joven, aparece una que cada vez va adquiriendo más importancia: el Trastorno Alimentario.

Estos trastornos implican una significativa alteración de la conducta, que afecta directamente la ingesta e implica acciones compensatorias para disminuir el peso corporal, todo motivado por el irrefrenable deseo de alcanzar la delgadez a toda costa (Franco, Díaz, López-Espinosa, Escoto, \& Camacho, 2013). A su vez, se presenta una evidente alteración de la imagen corporal (Moreno González \& Ortiz Viveros, 2009) acompañada de cambios en el carácter, retraimiento social y rasgos depresivos. Entre los trastornos más comunes se encuentran: la Anorexia, la Bulimia y el Trastorno Alimentario No Especificado (TANE), en todos ellos, más del 90\% de los casos ocurren en mujeres (American Psychiatric Association, 1994) y la mayoría padecen otros trastornos psiquiátricos comórbidos que no reciben el tratamiento indicado (Benjet, Méndez, Borges, \& Medina-Mora, 2012).

Para el diagnóstico de la anorexia, el Manual diagnóstico y estadístico de los trastornos mentales (American Psychiatric Association, 1994) define los siguientes síntomas: peso corporal por debajo del nivel normal mínimo esperado para su edad y talla, miedo intenso a ganar peso o a desarrollar obesidad, alteración de la percepción de la silueta corporal, presencia de irregularidades en el ciclo menstrual y eventualmente amenorrea en adolescentes post puberales. En el diagnóstico de la bulimia, se identifican atracones recurrentes y conductas compensatorias inapropiadas de manera repetida, con el fin de no ganar peso y mantener cierta silueta corporal (American Psychiatric Association, 1994). El TANE se refiere a un trastorno de la 
conducta alimentaria que no cumple todos los criterios para la Anorexia ni para la Bulimia (por ejemplo, disminución de peso, pero estando dentro de los límites de normalidad). Se estima que el TANE puede representar más de la mitad del total de los trastornos alimentarios (Leija, Sauceda, \& Ulloa, 2011). Las estrategias utilizadas para perder peso corporal consisten en una disminución de la ingesta total, utilización de purgas y práctica excesiva de ejercicio, los cuales constituyen elementos adversos para el tratamiento y la rehabilitación. Quienes presentan algún trastorno alimentario y utilizan la actividad física como estrategia compensatoria, es muy probable que hayan desarrollado una obsesión y cierto grado de adicción. Incluso la práctica deportiva de alto rendimiento conlleva una preocupación por la estética y la imagen corporal que puede derivar en síntomas de Trastornos alimenticios (Dosil, 2008). Si el Trastorno Alimenticio aún no requiere hospitalización, la actividad física prescrita conjuntamente (médico, psicológico, nutricional) podría obtener resultados positivos y saludables a nivel físico y mental (De la Cruz, Moreno, Pino, \& Martínez, 2011; Dunn, Trivedi, Kampert, Clark, \& Chambliss, 2005; Palushka \& Schwenk, 2000; Tolmunen et al., 2006), reduciendo la ansiedad y la depresión, y aumentando la socialización (Weinberg \& Gould, 1996).

De entre los conocimientos teóricos y empíricos traspasados de la psicología al ámbito del deporte está el entrenamiento mental, que consiste en dotar al deportista de estrategias mentales que mejoren su rendimiento y se desarrollen como personas (por ejemplo, establecimiento de objetivos, la visualización, la relajación progresiva y el control de pensamientos). Una interesante propuesta de intervención psicológica relacionada con trastornos alimentarios es la de Olmedilla y Andreu (2002) que proponen el auto-registro en el trabajo directo con el deportista y la participación de todo su entorno social inmediato. No se ha encontrado intervenciones concretas que usaran el entrenamiento mental para ayudar a desórdenes alimenticios y hábitos de excesiva práctica física. Los antecedentes más similares se reducen a dos estudios (Korrelboom, de Jong, Huijbrechts, \& Daansen, 2009; Kusel, 1999). El primero consiguió disminuir las conductas no saludables respecto a la alimentación de chicas de primaria usando un tratamiento de entrenamiento multimedia de vídeos y discusión de dos días de duración, incluyendo la interiorización del estereotipo corporal, aumento de la autoestima y percepción de realismo de las circunstancias que provocan los desórdenes. El segundo estudio también aplicó en pacientes holandesas con desórdenes alimenticios un programa que incluía trabajar la imagen corporal, entre otras estrategias, con técnicas de imaginación de escenas personales positivas. De esta manera lograron incrementar su autoestima y disminuir el estado depresivo. Diversos estudios, reforzando el tratamiento que hemos usado en nuestra investigación, han concluido que se precisan intervenciones de entrenamiento mental para solucionar trastornos alimenticios (Carr, 2006; Lloyd \& Foster, 2006).

Consecuentemente, el objetivo del presente estudio fue examinar el efecto de un programa de entrenamiento mental breve sobre el estado afectivo, las actitudes hacia la alimentación y los niveles de actividad física en adolescentes diagnosticadas con TANE que presentaban hábitos exigentes de actividad física.

\section{Metodología}

\section{Participantes}

El protocolo del presente estudio fue primero aprobado por el Comité de Evaluación Ético científico del Servicio de Salud [eliminado por anonimato]. Además, se ha respetado los acuerdos de la Declaración de Helsinki en su revisión de octubre del año 2013, elaborada por la Asociación Médica Mundial. A continuación, se invitó a participar a las adolescentes que fueron diagnosticadas con TANE en un grupo de Centros de Atención en Salud de la Ciudad de Valparaíso (Chile) en los últimos seis meses. Se informo ampliamente a todas las adolescentes y a sus tutores legales sobre las características del estudio. Antes de 
participar en el presente estudio se obtuvo por escrito el asentimiento informado de las adolescentes y el consentimiento informado de sus madres, padres o tutores legales. De todas ellas (37 adolescentes), 17 adolescentes con edades entre los 15 y 17 años (media $=16,13 \pm 0,64$ ) aceptaron participar en el presente estudio. Luego de las 17, solo ocho cumplieron con los criterios de inclusión y, por tanto, recibió el programa.

Los criterios de inclusión fueron: (1) Tener una pérdida de peso evidente, aunque dentro de los límites normales en relación a la edad y talla; (2) Presentar menstruaciones regulares; (3) Asistir a algunos de los gimnasios de la comuna; (4) Practicar una rutina de actividad física de al menos 90 minutos continuos cuatro veces por semana (que sin ser inapropiado para jóvenes sanas, sí lo sería considerando el diagnóstico de las participantes); (5) Estar recibiendo tratamiento farmacológico o psicológico; (6) Señalar en la entrevista inicial como motivos para la práctica de actividad física aspectos relacionados con el entorno social como factor determinante e influyente en sus hábitos; (7) Presentar el consentimiento informado firmado por sus padres, madres o tutores legales, y (8) presentar el asentimiento informado por parte de los escolares. En cuanto a los criterios de exclusión fueron: (1) No participar en todas las sesiones del programa de intervención, y (2) No cumplimentar correctamente todas las variables dependientes. Las ocho adolescentes cumplieron satisfactoriamente con los criterios de exclusión.

\section{Medidas}

Estado afectivo. Se aplicó la versión española del Inventario de Depresión de Beck (IDB) (Beck y Ward, 1961), que consta de 21 ítems orientados a medir la sintomatología característica del cuadro. Las afirmaciones de cada ítem son categorizadas para reflejar el grado de severidad del síntoma, desde su ausencia a su máxima expresión, para lo cual fueron graduadas de 0 a 3 puntos. La puntuación total fluctúa entre 0 y 63 , correspondiendo a mayor puntuación una mayor sintomatología depresiva. La versión española del IDB mostró adecuadas propiedades psicométricas entre los adolescentes (Alfa de Cronbach $=0,92 ; p<0,001$ ) (Beltrán, Freyre, \& Hernández-Guzmán, 2012).

Actitudes ante la alimentación. Se utilizó la versión española del cuestionario de actitudes ante la alimentación (EAT-26) (Garner, Olmsted, Bohr, \& Garfinkel, 1982), el que consta de 26 ítems cuyo objetivo es identificar la predisposición psicológica de presentar un trastorno alimentario, reflejando la relación que el sujeto tiene con la comida. Las afirmaciones poseen seis opciones de respuesta: siempre, casi siempre, frecuentemente, a veces, rara vez y nunca. Las preguntas desde la 1 a la 24 y la 26 se puntúan de la siguiente manera: nunca, rara vez y a veces 0 puntos, frecuentemente 1 punto, casi siempre 2 puntos y siempre 3 puntos. Para el caso del ítem 25, la puntuación es inversa. La versión española del EAT-26 mostró adecuadas propiedades psicométricas (Alfa de Cronbach =0,90; $p<0,001$ ) (Rivas, Bersabé, Jiménez, \& Berrocal, 2010).

Actividad física. Se elaboró un protocolo de entrevista semi-estructurada con tres temas o ítems que resultaron después de un proceso de validación por 12 jueces expertos, entre los que se encontraban psicólogos, médicos, docentes de educación física, entrenadores físicos y nutricionistas. Los ítems resultantes fueron: (1) ¿Cuántas sesiones por semana realiza actividad física?, (2) Cuando realiza actividad física, ¿cuánto tiempo dedica a cada práctica? y (3) Señale los motivos que tiene para practicar actividad física.

Lo anterior se obtuvo luego de presentar el estudio y sus objetivos a cada uno de los expertos, solicitando que identificaran los datos fundamentales respecto de los hábitos de práctica de actividad física que deben obtenerse en una entrevista inicial con las participantes. En un primer momento, cada experto entregó una propuesta libre de ítems que apuntaban a cuestiones centrales y específicas según su especialidad. Posteriormente se efectuó una reunión conjunta con todos los expertos, se discutió al respecto y se formularon los tres ítems finales. 


\section{Procedimiento}

Debido a la limitación ética de no realizar el tratamiento con todos las participantes, el presente estudio siguió un diseño preexperimental pretest-postest (Ato, López, \& Benavente, 2013). El protocolo que se siguió consistió en contactar a las autoridades de los centros de salud de la red asistencial de Valparaíso, explicar los alcances del estudio, y ofrecernos para trabajar con las pacientes que se ajustaran a los objetivos de éste. En los centros que aceptaron la propuesta, se procedió a revisar los ingresos y diagnósticos diarios de los últimos seis meses, encontrando un total de 37 casos potenciales. Se citó a cada una de las participantes potenciales a una entrevista de evaluación, constatando que 17 cumplían con los criterios de inclusión fijados para este estudio. A estas últimas se les habló acerca del programa y se les citó a una segunda entrevista acompañada de alguno de sus padres, donde a cada una de ellas se les aplicaron las pruebas de medición. Finalmente, se contó con el consentimiento para trabajar con ocho adolescentes a las que una semana después se les comenzó a aplicar de programa.

El entrenamiento mental breve consistió en un programa de intervención psicológica de ocho sesiones, orientado a reencuadrar las actitudes que presentaban los sujetos tanto hacia sí mismo como hacia la comida y la actividad física. El programa tiene un enfoque cognitivo de corte integrativo, enfatizando los aspectos actitudinales, los valores, y los aspectos emocionales y conductuales. Su construcción tomó como base, entre otros, un modelo de intervención cognitivo conductual para la depresión enmascarada (de la Cerda \& de la Maza, 1997) y un programa de intervención escolar para la prevención primaria de trastornos alimentarios (Ruiz-Lázaro, 2000). Las sesiones, todas ellas grupales y desarrolladas bajo la modalidad taller, se distribuyeron en ocho semanas (una sesión de 2 horas de duración por semana) de la siguiente forma:

Sesión $n^{0} 1$. Conocimiento y establecimiento de objetivos. Orientada a enfocar las metas y alcances del programa, delinear expectativas, facilitar el conocimiento grupal y establecer la confianza básica necesaria para impulsar el aprendizaje individual y colectivo. Las técnicas utilizadas fueron: dinámica de presentación individual y presentación de los objetivos del taller y cronograma de trabajo (30 minutos), exposición individual de experiencias relacionadas con la imagen corporal y la conducta alimentaria ( 40 minutos), charla sobre nutrición y hábitos de vida saludable (20 minutos), reflexión grupal acerca de la imagen corporal y su importancia en el autoconcepto y bienestar psicológico (30 minutos).

Sesión $n^{\circ} 2$. Focalización y toma de conciencia mental y corporal. Orientada a propiciar el autoconocimiento, la autoaceptación y reconocimiento de las propias señales mentales y corporales a la base de los hábitos y comportamientos. Las técnicas utilizadas fueron: presentación individual del yo real y el yo ideal (30 minutos), el árbol de mi autoestima (30 minutos), ejercicios individuales y grupales de imaginería y captación de ritmos biológicos y sensaciones corporales sentidas ( 40 minutos), reflexión grupal acerca del papel de la mente, el pensamiento y la inteligencia en el bienestar psicológico (20 minutos).

Sesión $n^{o}$ 3. Autocontrol psicobiológico. Orientada a propiciar el control emocional y automanejo sintomático mediante el aprendizaje y práctica de estrategias de relajación corporal y visualización cognitiva. Reinterpretación de imágenes pasadas, presentes y futuras, orientándolas al logro de bienestar físico y emocional. Las técnicas utilizadas fueron: entrenamiento en relajación progresiva (20 minutos), entrenamiento autógeno (20 minutos), reflexión grupal y conclusiones (20 minutos), visualización (15 minutos), modelado encubierto (15 minutos), reflexión final y conclusiones (30 minutos).

Sesión $n^{\circ} 4$. Reposicionamiento del locus de control. Orientada a re-significar el valor de la propia voluntad y del sentido del yo como principal agente de cambio para el logro del bienestar personal. Las técnicas utilizadas fueron: análisis individual de experiencias recientes relacionadas con los hábitos alimenticios y la actividad física (30 minutos), explicación de los conceptos de atribución y locus de control y ejercitación en base a experiencias personales (30 minutos), reorientación de las experiencias analizadas considerando un locus de control interno y expectativas de autoeficacia adecuadas (30 minutos), reflexión autoevaluativa individual 
y grupal en relación a lo trabajado y logros obtenidos hasta el momento, primera mitad del programa (30 minutos).

Sesión $n^{0}$ 5. Autogestión y control de los pensamientos. Orientada a la identificación de pensamientos facilitadores y perturbadores relacionados con los hábitos alimenticios y otros, alineación de dimensiones cognitivas, emocionales y conductuales. Las técnicas utilizadas fueron: exposición de modelo psicológico cognitivo conductual, enfatizando la importancia de los pensamientos en las respuestas emocionales y conductuales (20 minutos), aplicación de ejercicios experienciales orientados a la identificación y reestructuración de pensamientos automáticos e ideas irracionales (40 minutos), aprendizaje de estrategias pragmáticas y aplicadas de reencuadre cognitivo y solución de problemas (40 minutos), reflexión final y conclusiones (20 minutos).

Sesión $n^{\circ}$ 6. Re-focalización y reencuadre motivacional (parte 1). Dirigida a la reorientación de prioridades y prácticas, análisis experiencial de la situación presente y sus perspectivas a futuro (corto, mediano y largo plazo) relacionado con los hábitos alimenticios y práctica de actividad física. Las técnicas utilizadas fueron: exposición de película "Malos hábitos" [1 $1^{\text {a }}$ parte], México, 2007 (60 minutos), reflexión y análisis (20 minutos), elaboración individual y asistida de línea de la vida futura, definiendo metas y estrategias de consecución a corto, mediano y largo plazo relacionada con los hábitos alimenticios y práctica de actividad física (20 minutos), exposición del plan personal ante el grupo y conclusiones (20 minutos).

Sesión $n^{\circ}$ 7. Re-focalización y reencuadre motivacional (parte 2). Dirigida al análisis de situaciones vitales posibles de enfrentar en distintos escenarios vitales: personales, escolares, afectivos y sociales. Orientación al logro de objetivos de autocuidado y crecimiento personal, potenciando la práctica de hábitos de vida saludables. Las técnicas utilizadas fueron: exposición de película "Malos hábitos" [2a parte], México, 2007 (60 minutos), reflexión y análisis (20 minutos), elaboración individual y asistida de la línea de la vida futura, definiendo metas y estrategias de consecución a corto, mediano y largo plazo relacionada con otros escenarios vitales (20 minutos), exposición del plan personal ante el grupo y conclusiones (20 minutos).

Sesión $n^{\circ}$ 8. Generalización y enfoque responsable de la voluntad. Orientada al reforzamiento de los principios y métodos aprendidos, a la retroalimentación respecto de lo vivido en las ocho sesiones y al establecimiento de compromiso personal de autocuidado. Las técnicas utilizadas fueron: debate reflexivo, análisis y valoración personal y grupal respecto de la participación en el programa (60 minutos), visualización y ensayo cognitivo respecto de "mi vida futura" (20 minutos), elaboración de una carta personal a familiares y seres queridos "Yo me comprometo con mi salud, me conozco, me quiero y me cuido" y posterior lectura de carta al resto del grupo (30 minutos), cierre y palabras motivacionales finales por parte del facilitador (10 minutos).

Después de una semana de haber finalizado el programa, se aplicaron nuevamente los instrumentos de medición, citando individualmente a cada participante. Con el fin de obtener datos comparativos, también se citó a las nueve adolescentes que no dieron su consentimiento para participar en el programa, sin embargo, ninguna de ellas finalmente asistió.

\section{Análisis estadístico}

Se realizó una estadística descriptiva (media \pm desviación estándar/ frecuencia y porcentaje) de todas las variables dependientes. Posteriormente, se aplicó la prueba de Wilcoxon para comparar los valores de las dos mediciones efectuadas con el IDB y el EAT-26 y para el nivel de actividad física (días a la semana y minutos de práctica de actividad física por sesión). Luego se analizó el contenido de la tercera pregunta de la entrevista correspondiente a motivos de práctica. Además de categorizar la aparición o no de cada motivo en cada participante, se tabuló tantas veces como se hubieran dicho, ya que consideramos que era un indicador de la importancia otorgada por las participantes a estos motivos de práctica que aportaba en sus respuestas. El 
tamaño de efecto se estimó mediante el valor $r$ (Field, 2017). De acuerdo con las directrices de Cohen (1992), se estableció valores de $r$ igual a $0,10-0,29$ como bajos, $0,30-0,49$ como moderados y 0,50-1,00 como altos. Los análisis estadísticos se realizaron mediante el programa estadístico SPSS versión 21.0 para Windows $\left(\right.$ SPSS $^{\bullet}$ Inc., Chicago, IL). El nivel de significación estadística se estableció en $\mathrm{p}<0,05$.

\section{RESULTADOS Y DISCUSIÓN}

La Tabla 1 muestra el efecto del programa de entrenamiento mental breve sobre el estado afectivo, actitudes ante la alimentación y niveles de actividad física. Los resultados de la prueba de Wilcoxon mostraron que el programa de intervención disminuyó estadísticamente los niveles de estado afectivo, actitudes ante la alimentación y niveles de actividad física $(\mathrm{p}<0,05)$. Además, el tamaño del efecto fue alto $(\# \mathrm{r} \#=0,63-0,65)$.

TABLA 1

Efecto del programa de entrenamiento mental breve sobre el estado afectivo, actitudes ante la alimentación y niveles de actividad física $(\mathrm{n}=8)$

\begin{tabular}{lccccc}
\hline & Pre-intervención & Post-intervención & Prueba de Wilcoxon & TE \\
\cline { 2 - 5 } Variables & Media \pm DE & Media \pm DE & $Z$ & $p$ & $r$ \\
\hline IDB & $22,25 \pm 4,10$ & $14,13 \pm 3,56$ & $-2,555$ & 0,011 & $-0,64$ \\
EAT-26 & $31,88 \pm 5,28$ & $24,38 \pm 4,96$ & $-2,536$ & 0,011 & $-0,63$ \\
Sesiones/semana & $4,25 \pm 0,89$ & $3,25 \pm 0,71$ & $-2,530$ & 0,011 & $-0,63$ \\
Minutos/sesión & $105,00 \pm 13,89$ & $82,50 \pm 16,04$ & $-2,585$ & 0,010 & $-0,65$ \\
\hline
\end{tabular}

Nota. $\mathrm{DE}=$ Desviación estándar; $\mathrm{TE}=$ Tamaño de efecto; $\mathrm{IDB}=$ Inventario de Depresión de Beck; EAT-26 = Test de actitudes ante la alimentación.

En relación a los motivos de la práctica de actividad física, los resultados se muestran en la Tabla 2. Antes de aplicado el programa las respuestas más frecuentes apuntaban a motivos que podemos considerar negativos (control del peso, 43\%; imagen corporal, 30\%). Sin embargo, después del programa la distribución de las respuestas fue más dispersa y hubo un notorio cambio en la tendencia hacia respuestas consideradas como positivas (salud, 23\%; estado de ánimo, 19\%; y relajación, 17\%).

Algunos ejemplos de respuesta de las categorías señaladas fueron las siguientes: Salud: "sentirme mejor físicamente", "cuidar mi cuerpo"; Estado de ánimo: "sentirme con más energía”, "estar menos triste”; Relajación: "controlar la ansiedad", "tranquilizarme, relajarme"; Autoestima: "aceptarme más", "sentirme mejor conmigo misma"; Imagen corporal: "verme más atractiva", "poder mirarme al espejo"; Control del peso: "bajar de peso", "estar más flaca”. 
TABLA 2

Número de participantes que aportaban cada motivo de práctica de actividad física y cantidad total en que los mencionaban (entre paréntesis) $(\mathrm{n}=8)$

\begin{tabular}{|c|c|c|c|c|}
\hline \multirow[b]{2}{*}{ Motivos de práctica } & \multicolumn{2}{|c|}{ Pre-intervención } & \multicolumn{2}{|c|}{ Post-intervención } \\
\hline & Frecuencia & Porcentajes & Frecuencia & Porcentajes \\
\hline Control del peso & $6(26)$ & $75,0(43,0)$ & $1(6)$ & $12,5(12,0)$ \\
\hline Imagen corporal & $4(18)$ & $50,0(30,0)$ & $1(7)$ & $12,5(14,5)$ \\
\hline Salud & $3(10)$ & $37,5(17,0)$ & $5(11)$ & $62,5(23,0)$ \\
\hline Relajación & $1(6)$ & $12,5(10,0)$ & $3(8)$ & $37,5(17,0)$ \\
\hline Esta do de ánimo & $0(0)$ & $0,0(0,0)$ & $3(9)$ & $37,5(19,0)$ \\
\hline Autoestima & $0(0)$ & $0,0(0,0)$ & $3(7)$ & $37,5(14,5)$ \\
\hline
\end{tabular}

En definitiva, los resultados mostraron la eficacia del programa disminuyendo significativamente la sintomatología depresiva, mejorando la actitud hacia la alimentación y mejorando los motivos y la práctica de actividad física en la muestra estudiada. De esta forma se rechaza la hipótesis nula y se confirma la efectividad del programa de entrenamiento mental breve, coincidiendo con la eficacia obtenida en los escasos estudios similares encontrados (Korrelboom et al., 2009; Kusel, 1999).

Lo anterior sugiere que es posible seguir practicando actividad física, siempre y cuando esté bien monitoreada y vaya re-encuadrándose el foco motivacional de base. En efecto, está demostrado que la actividad física se relaciona directamente con la remisión y el control de psicopatologías (Dunn et al., 2005; Palushka \& Schwenk, 2000; Tolmunen et al., 2006). Por tanto, considerando todas las variables sanitarias implicadas, es posible considerarla como una aliada en el tratamiento de estos casos, más aún si se enmarca en un contexto de entrenamiento mental adaptado especialmente a las características de la muestra y utilizando métodos y estrategias recomendadas por los especialistas (Dosil, 2008).

Estos resultados también podrían explicarse debido a que en esta modalidad, además de un efecto a nivel psicológico-emocional y conductual, se cumpliría lo planteado en la tesis relativa al ocio, donde el programa aplicado funcionó como elemento favorable para la socialización y permitió la vivencia de experiencias significativas, así como una retroalimentación real y genuina para las participantes (Weinberg \& Gould, 1996). En el grupo, las participantes encontraron otras personas con inquietudes y sentires similares, todas ellas dispuestas a vivir nuevas experiencias, donde lo social y afectivo jugó un rol fundamental para mejorar su estado afectivo.

No obstante, las mejorías no fueron completas, las participantes obtuvieron puntuaciones equivalentes al rango de depresión leve y reflejaron un cierto nivel de riesgo en las actitudes hacia la alimentación. Es probable que esto se relacione con la duración de las sesiones del programa, ante lo cual resultaría interesante profundizar esta línea de investigación proponiendo futuros estudios que impliquen una intervención más prolongada para constatar si es posible una reducción sintomática y mejoría mayores.

$\mathrm{Al}$ mismo tiempo reconocemos el tamaño de la muestra como limitación del estudio, lo cual acota las conclusiones a la muestra específica que formó parte de esta investigación. En efecto, sujetos que cumplan con 
criterios de inclusión aplicados en este estudio son escasos, aunque debemos tener en cuenta que el estudio contó primeramente con 37 participantes y se intentó intervenir con 17. Al mismo tiempo, resaltamos que no hay estudios previos que relacionen directamente todas las variables consideradas en esta investigación.

Finalmente, considerando todo lo anterior, estamos en condiciones de sugerir la aplicación del presente entrenamiento mental breve para mejorar el estado afectivo, las actitudes hacia la alimentación y la percepción de la actividad física en adolescentes diagnosticadas con TANE que practiquen una rutina exigente de actividad física.

\section{Conclusiones}

Un programa de entrenamiento mental breve, de ocho sesiones de duración distribuidas en ocho semanas, es efectivo disminuyendo la sintomatología depresiva, mejorando la actitud hacia la alimentación y mejorando los motivos y la práctica de actividad física en adolescentes diagnosticadas con TANE y aficionadas a rutinas exigentes de actividad física. Por tanto, un programa de entrenamiento mental breve sería recomendable como complemento terapéutico en adolescentes con tales características.

\section{Agradecimientos}

Los autores agradecemos a los directores de los Centros de Salud participantes por permitir la puesta en práctica de este estudio otorgando todas las facilidades logísticas y estructurales para ello.

\section{ReFERENCIAS}

American Psychiatric Association (1994). Diagnostic and statistical manual of mental disorders (4th ed). Washington: Masson.

Ato, M., López, J. J., y Benavente, A. (2013). Un sistema de clasificación de los diseños de investigación en psicología. Anales de Psicología, 29, 1038-1059. http://dx.doi.org/10.6018/analesps.29.3.178511

Beck, A., Ward, C., Mendelson, M., Mock, J., y Erbaugh, J. (1961). An inventory for measuring depression. Archives of General Psychiatry, 4, 561-571. https://www.ncbi.nlm.nih.gov/pubmed/13688369

Behar, R. (1996). El perfeccionismo en los trastornos del hábito del comer. Revista Chilena de Neuro-Psiquiatría, 34, 257-262.

Beltrán, M. C., Freyre, M. A., y Hernández-Guzmán, L. (2012). El Inventario de Depresión de Beck: Su validez en población adolescente. Terapia Psicológica, 30, 5-13. http://dx.doi.org/10.4067/S0718-48082012000100001

Benjet, C., Méndez, E., Borges, G., y Medina-Mora, M. (2012). Epidemiología de los trastornos de la conducta alimentaria en una muestra representativa de adolescentes. Salud Mental, 35, 483-490. http:// www.scielo.org.mx/pdf/sm/v35n6/v35n6a5.pdf

Carr, C. M. (2006). Sport psychology: Psychologic issues and applications. Physical Medicine and Rehabilitation Clinics of North America, 17, 519-535. https://doi.org/10.1016/j.pmr.2006.05.007

Cohen, J. (1992). A power primer. Psychology Bulletin, 112(1), 155-159. https://www.ncbi.nlm.nih.gov/ pubmed/19565683

De la Cerda, P., y de la Maza R. (1997). Aplicación y evaluación de un modelo de intervención cognitivo-conductual en casos de depresión enmascarada (Tesis doctoral). Universidad de Valparaíso, Chile.

De la Cruz, E., Moreno, M., Pino, J., y Martínez, R. (2011). Actividad física durante el tiempo libre y su relación con algunos indicadores de salud mental en España. Salud Mental, 34, 45-52. http://www.scielo.org.mx/pdf/sm/ v34n1/v34n1a6.pdf 
Delgado, M., Gutiérrez, A., y Castillo, M. (2004). Entrenamiento fisico-deportivo y alimentación. De la infancia a la edad adulta. Barcelona: Paidotribo.

Dosil, J. (2008). Psicología de la actividad fisica y del deporte (2nd ed.). Madrid: Mc Graw Hill.

Dunn, A. L., Trivedi, M. H., Kampert, J. B., Clark, C. G., y Chambliss, H. O. (2005). Exercise treatment for depressionefficacy and dose response. American Journal of Preventive Medicine, 28, 1-8. https://doi.org/10.1016/ j.amepre.2004.09.003

Field, A. (2017). Discovering statistics using IBM SPSS Statistics (4th ed.). London, UK: SAGE.

Franco, K., Díaz, F. J., López-Espinosa, A., Escoto, M. D. C. \& Camacho, E. J. (2013). Variables predictoras de riesgo de trastorno del comportamiento alimentario en mujeres. Terapia Psicológica, 31, 219-225. http://www.scielo.cl/ $\mathrm{pdf} /$ terpsicol/v31n2/art08.pdf

Garner, D. M., Olmsted, M. P., Bohr, Y., y Garfinkel, P. E. (1982). The Eating Attitudes Test: Psychometric features and clinical correlates. Psychological Medicine, 12, 871-878. https://www.ncbi.nlm.nih.gov/pubmed/6961471

Harter, S. (2003). The construction and conservation of the self: James and Cooley revisited. En Coleman, J. C. y Hendry, L. B. (eds.). Psicología de la adolescencia (pp. 43-70). Madrid: Ediciones Morata SL.

Korrelboom, K., de Jong, M., Huijbrechts, I., y Daansen, P. (2009). Competitive memory training (COMET) for treating low self-esteem in patients with eating disorders: A randomized clinical trial.Journal of Consulting and Clinical Psychology, 77, 974-980. https://doi.org/10.1037/a0016742

Kusel, A. B. (1999). Primary prevention of eating disorders through media literacy training of girls. Dissertation abstracts international: Section B: The sciences and engineering, 60(4-B), 1859.

Leija, M., Sauceda, J., y Ulloa, R. (2011). Características familiares y trastornos de la conducta alimentaria en una muestra de mujeres adolescentes internadas en un hospital psiquiátrico. Salud Mental, 34, 203-210. http:// www.scielo.org.mx/pdf/sm/v34n3/v34n3a3.pdf

Lloyd, P. J., y Foster, S. L. (2006). Creating healthy, high-performance workplaces: Strategies from health and sports psychology. Consulting Psychology Journal: Practice and Research, 58, 23-39. https:// doi.org/10.1037/1065-9293.58.1.23

Ministerio de Salud (2005). Estrategia Global contra la Obesidad EGO Chile. Recuperado en http://web.minsal.cl/ estr_global_obesidad

Moreno González, M. A., y Ortiz Viveros, G. R. (2009). Trastorno alimentario y su relación con la imagen corporal y la autoestima en adolescentes. Terapia Psicológica, 27, 181-190. http://dx.doi.org/10.4067/ S0718-48082009000200004

Olmedilla, A., y Andreu, M. (2002). Propuesta de intervención psicológica para el control de hábitos alimentarios en deportistas jóvenes. Cuadernos de Psicología del Deporte, 2, 13-28. http://revistas.um.es/cpd/article/ view/111781/106101

Palushka, S. A., y Schwenk, T. L. (2000). Physical activity and mental health: Current concepts. Sports Medicine, 29, 167-180. https://www.ncbi.nlm.nih.gov/pubmed/10739267

Rivas, T., Bersabé, R., Jiménez, M., y Berrocal, C. (2010). The Eating Attitudes Test (EAT-26): Reliability and validity in Spanish female samples. The Spanish Journal of Psycholy, 13, 1044-1056. https://www.ncbi.nlm.nih.gov/ pubmed/20977051

Ruiz-Lázaro, P. M. (2000). Prevención de los trastornos de la conducta alimentaria. Revista de Psiquiatría InfantoJuvenil, 1, 18-31. https://www.ncbi.nlm.nih.gov/pubmed/20977051

Tolmunen, T., Laukkanen, J., Hintikka, J., Kurl, S., Viinamäki, H., Salonen, R.,... Salonen, J. T. (2006). Low maximal oxygen uptake is associated with elevated depressive symptoms in middle-aged men. European Journal of Epidemiology, 21, 701-706. http://dx.doi.org/10.1007/s10654-006-9038-5

Vásquez, C., de Cos, A. I., y López-Nomdedeu, C. (2005). Alimentación y nutrición, manual teórico-práctico. Madrid: Ediciones Díaz de Santos.

Weinberg, R., y Gould, D. (1996). Fundamentos de psicología del deporte y el ejercicio físico. Barcelona: Ariel. 